\title{
Origin of strong magnetic fields in Milky Way-like galaxies
}

\author{
Alexander M. Beck \\ University Observatory Munich \\ Scheinerstr. 1, D-81679 Munich, Germany \\ email: abeck@usm.lmu.de
}

\begin{abstract}
Magnetic fields are observed on all scales in the Universe (see e.g. Kronberg 1994), but little is known about the origin and evolution of those fields with cosmic time. Seed fields of arbitrary source must be amplified to present-day values and distributed among cosmic structures. Therefore, the emergence of cosmic magnetic fields and corresponding dynamo processes (see e.g. Zel'dovich et al. 1983; Kulsrud et al. 1997) can only be jointly understood with the very basic processes of structure and galaxy formation (see e.g. Mo et al. 2010).
\end{abstract}

Keywords. methods: numerical, galaxies: formation, galaxies: magnetic fields, early Universe.

\section{Models and simulations of the magnetic field growth}

The magnetic field is described by the induction equation of magnetohydrodynamics

$$
\frac{\partial \mathbf{B}}{\partial t}=\nabla \times[\mathbf{v} \times \mathbf{B}-\eta \nabla \times \mathbf{B}]+\left.\frac{\partial \mathbf{B}}{\partial t}\right|_{\text {seed }},
$$

where the description of astrophysical processes enters via the velocity field $\mathbf{v}$ and the plasma microphysics via the resistivity $\eta$. Furthermore, a magnetic seed field is required.

The dashed lines in Figure 1 show the analytical model for the primordial field growth (see Beck et al. 2012, for details) during galaxy formation with a solution of

$$
B(a)=\frac{1}{a^{2}}\left[\left(4 \pi \rho v_{\mathrm{turb}}^{2}\right)^{-1}+B_{0}^{-2} e^{-2 \gamma t(a)}\right]^{-\frac{1}{2}},
$$

where $a$ is the cosmological scale-factor, $\gamma$ the growth rate, $B_{0}$ the initial field amplitude and $1 / 2 \rho v_{\text {turb }}^{2}$ the turbulent pressure as saturation level.

Another possibility is to create magnetic seed fields within the very first star forming objects by supernovae. This approach proposed by Rees (1987) and simulated by Beck et al. (2013a) gives very promising results. The dotted line in Figure 1 shows the contribution resulting only from supernovae without subsequent amplification within a Milky Way-like galactic halo. Given typical properties of the interstellar medium it approximates to

$$
\dot{B}_{\text {seed }} \approx B_{\mathrm{SN}}\left(\frac{r_{\mathrm{SN}}}{r_{\mathrm{SB}}}\right)^{2}\left(\frac{r_{\mathrm{SB}}}{r_{\mathrm{Inj}}}\right)^{3} \frac{\sqrt{\dot{N}_{\mathrm{SN}} \Delta t}}{\Delta t} \approx 10^{-9} \frac{\mathrm{G}}{\mathrm{Gyr}} .
$$

The solid line in Figure 1 shows the results of the numerical simulations of Milky Way-like halo formation including magnetic fields of supernova origin. The simulations are based on the GADGET-3 code (Springel 2005) and a simple prescription for star formation (Springel \& Hernquist 2003). We conclude that the magnetic fields of galaxies can be described as a result of the very basic processes of star and galaxy formation. The seed fields can be supplied by supernovae and the formation of structures yields 


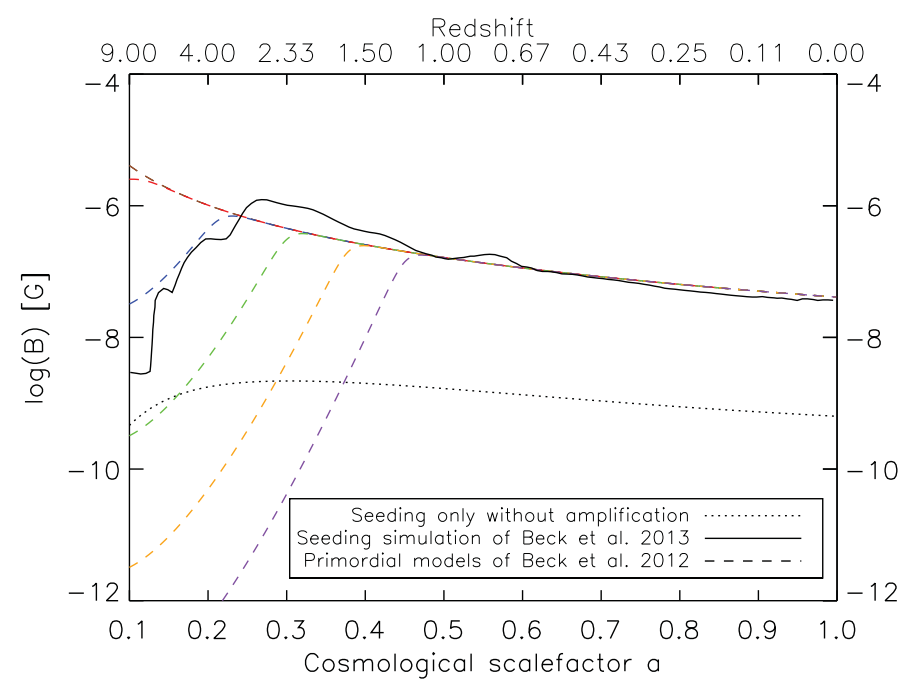

Figure 1. Mean amplitude of the magnetic field versus cosmic time composed from the analytical models of Beck et al. (2012) and numerical simulations of Beck et al. (2013a).

sufficient dynamo action to explain strong field amplitudes. However, with magnetic fields only present within collapsing and virialising structures, the question now arises how these fields can be transported into the large-scale structure and possible voids (Beck et al. 2013b).

\section{Acknowledgments}

This work is supported by the DFG Cluster of Excellence 'Universe', DFG Research Unit '1254' and by the 'Magneticum' project (http://www.magneticum.org).

\section{References}

Beck, A. M., et al., 2012, MNRAS, 422, 2152

Beck, A. M., et al., 2013a, MNRAS, 435, 3575

Beck, A. M., et al., 2013b, MNRAS, 429, L60

Kronberg, P. P., 1994, Reports on Progress in Physics, 57, 325

Kulsrud, R. M., et al., 1997, ApJ, 480, 481

Mo, H., et al., 2010, Galaxy Formation and Evolution, Cambridge University Press

Rees, M. J., 1987, QJRAS, 28, 197

Springel, V. \& Hernquist L., 2003, MNRAS, 339, 289

Springel V., 2005, MNRAS, 364, 1105

Zel'dovich, Y. B., et al., 1983, Magnetic Fields in Astrophysics, Gordon and Breach, New York 